\title{
Exploring Prime Numbers and Modular Functions II: On the Prime Number via Elliptic Integral Function
}

\author{
Edigles Guedes ${ }^{1}$ and Prof. Dr. K. Raja Rama Gandhi ${ }^{2}$ \\ Number Theorist, Brazil ${ }^{1}$
}

Resource perosn in Mathematics for Oxford University Press and Professor at BITS-Vizag ${ }^{2}$

ABSTRACT. The main goal this paper is to develop an asymptotic formula for the prime number, using elliptic integral function.

\section{INTRODUCTION}

As consequence of the prime number theorem, I put the asymptotic formula for the $n$th prime number, denoted by $p_{n}$ :

$$
p_{n} \sim n \ln n \text {. }
$$

In this paper, I prove that

$$
p_{n} \sim 2 n K\left(\frac{256 n-4}{256 n+4}\right)-\frac{\ln 2}{8}(64 n+1)-\frac{\ln n}{64} .
$$

\section{THEOREM}

THEOREM 1. I have

$$
p_{n} \sim 2 n K\left(\frac{256 n-4}{256 n+4}\right)-\frac{\ln 2}{8}(64 n+1)-\frac{\ln n}{64}
$$

where $p_{n}$ denotes the $n$th prime number and $K(x)$ denotes the complete elliptic integral of first kind.

Proof. In [1], we encounter the identity

$$
\operatorname{agm}(x, y)=\frac{\pi}{4} \cdot \frac{x+y}{K\left(\frac{x-y}{x+y}\right)}
$$

In [2], we encounter an alternative for extremely high precision calculation is the formula

$$
\ln n \approx \frac{\pi}{2 \operatorname{agm}(1,4 / s)}-m \ln 2,
$$

where agm denotes the arithmetic-geometric mean of 1 and $4 / s$, and

$$
s=n \cdot 2^{m}>2^{p / 2},
$$

with $m$ chosen so that $p$ bits of precision is attained. Now, the value of 8 for $m$ is sufficient. Hence,

$$
\ln n \approx \frac{\pi}{2 \operatorname{agm}(1,4 / 256 n)}-8 \ln 2 .
$$

Substituting (2) into (3), I get around

$$
\ln n \approx \frac{512 n K\left(\frac{256 n-4}{256 n+4}\right)}{256 n+4}-8 \ln 2=\frac{128 n K\left(\frac{256 n-4}{256 n+4}\right)-8 \ln 2(64 n+1)}{64 n+1} .
$$


Wherefore,

$$
\begin{gathered}
(64 n+1) \ln n \approx 128 n K\left(\frac{256 n-4}{256 n+4}\right)-8 \ln 2(64 n+1) \\
n \ln n \approx \frac{128}{64} n K\left(\frac{256 n-4}{256 n+4}\right)-\frac{8}{64} \ln 2(64 n+1)-\frac{\ln n}{64} \\
n \ln n \approx 2 n K\left(\frac{256 n-4}{256 n+4}\right)-\frac{\ln 2}{8}(64 n+1)-\frac{\ln n}{64}
\end{gathered}
$$

I take (5) in (1), and achieve

$$
p_{n} \sim 2 n K\left(\frac{256 n-4}{256 n+4}\right)-\frac{\ln 2}{8}(64 n+1)-\frac{\ln n}{64} .
$$

This completes the proof. $\square$

\section{REFERENCES}

[1] http://en.wikipedia.org/wiki/Arithmetic_geometric_mean, available in November 16, 2013.

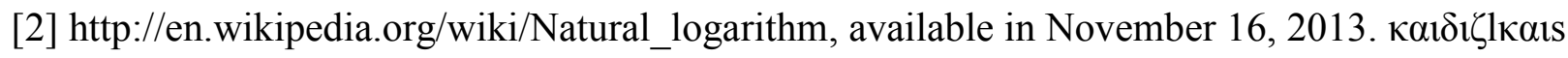

